\title{
On Lines and Joints
}

\section{Haim Kaplan • Micha Sharir • Eugenii Shustin}

Received: 2 June 2009 / Revised: 17 August 2009 / Accepted: 1 December 2009 /

Published online: 12 February 2010

(C) Springer Science+Business Media, LLC 2010

\begin{abstract}
Let $L$ be a set of $n$ lines in $\mathbb{R}^{d}$, for $d \geq 3$. A joint of $L$ is a point incident to at least $d$ lines of $L$, not all in a common hyperplane. Using a very simple algebraic proof technique, we show that the maximum possible number of joints of $L$ is $\Theta\left(n^{d /(d-1)}\right)$. For $d=3$, this is a considerable simplification of the original algebraic proof of Guth and Katz (Algebraic methods in discrete analogs of the Kakeya problem, 4 December 2008, arXiv:0812.1043), and of the follow-up simpler proof of Elekes et al. (On lines, joints, and incidences in three dimensions. Manuscript, 11 May 2009, arXiv:0905.1583). Some extensions, e.g., to the case of joints of algebraic curves, are also presented.
\end{abstract}

Keywords Joints · Arrangements of lines in space - Kakeya problem · Algebraic methods

Let $L$ be a set of $n$ lines in $\mathbb{R}^{d}$, for $d \geq 3$. A joint of $L$ is a point incident to at least $d$ lines of $L$, not all in a common hyperplane.

Work on this paper has been partly supported by the Hermann Minkowski-MINERVA Center for Geometry at Tel Aviv University. Work by Micha Sharir was also supported by NSF Grants CCF-05-14079 and CCF-08-30272, by Grant 155/05 from the Israel Science Fund. and by Grant 2006/194 from the U.S.-Israeli Binational Science Foundation. Work by Haim Kaplan was also supported by Grant 975/06 from the Israel Science Fund, and by Grant 2006/204 from the U.S.-Israel Binational Science Foundation.

H. Kaplan $(\bowtie) \cdot$ M. Sharir

School of Computer Science, Tel Aviv University, Tel Aviv 69978, Israel

e-mail: haimk@post.tau.ac.il

M. Sharir

Courant Institute of Mathematical Sciences, New York University, New York, NY 10012, USA

e-mail: michas@post.tau.ac.il

E. Shustin

School of Mathematical Sciences, Tel Aviv University, Tel Aviv 69978, Israel

e-mail: shustin@post.tau.ac.il 
A simple construction, using the axis-parallel lines in a $k \times k \times \cdots \times k$ grid, for $k=\Theta\left(n^{1 /(d-1)}\right)$, has $d k^{d-1}=\Theta(n)$ lines and $k^{d}=\Theta\left(n^{d /(d-1)}\right)$ joints.

In this paper we prove that this is a general upper bound. That is:

Theorem 1 The maximum possible number of joints in a set of $n$ lines in $\mathbb{R}^{d}$ is $\Theta\left(n^{d /(d-1)}\right)$.

Background The problem of bounding the number of joints, for the 3-dimensional case, has been around for almost 20 years [3, 7, 11] (see also [2, Chap. 7.1, Problem 4]), and, until very recently, the best-known upper bound, established by Sharir and Feldman [7], was $O\left(n^{1.6232}\right)$. The proof techniques were rather complicated, involving a battery of tools from combinatorial geometry, including forbidden subgraphs in extremal graph theory, space decomposition techniques, and some basic results in the geometry of lines in space (e.g., Plücker coordinates).

Wolff [12] observed a connection between the problem of counting joints to the Kakeya problem. Bennett et al. [1] exploited this connection and proved an upper bound on the number of so-called $\theta$-transverse joints in $\mathbb{R}^{3}$, namely, joints incident to at least one triple of lines for which the volume of the parallelepiped generated by the three unit vectors along these lines is at least $\theta$. This bound is $O\left(n^{3 / 2+\varepsilon} / \theta^{1 / 2+\varepsilon}\right)$, for any $\varepsilon>0$, where the constant of proportionality depends on $\varepsilon$.

It has long been conjectured that the correct upper bound on the number of joints (in three dimensions) is $O\left(n^{3 / 2}\right)$, matching the lower bound just noted. In a rather dramatic recent development, building on a recent result of Dvir [5] for a variant of the problem for finite fields, Guth and Katz [9] have settled the conjecture in the affirmative, showing that the number of joints (in three dimensions) is indeed $O\left(n^{3 / 2}\right)$. Their proof technique is completely different, and uses fairly simple tools from algebraic geometry. In a follow-up paper by Elekes et al. [6], the proof has been further simplified, and extended (a) to obtain bounds on the number of incidences between $n$ lines and (some of) their joints, and (b) to handle also flat points, which are points incident to at least three lines, all coplanar.

As far as we know, the problem has not yet been studied for $d>3$.

In this paper we give a very simple and short proof of Theorem 1; that is, we obtain a tight bound for the maximum possible number of joints in any dimension. The proof uses an algebraic approach similar to that of the other proofs, but is much simpler, shorter and more direct.

We also present several extensions of the result. One of them yields the same upper bound on the number of joints in a collection of algebraic curves in $\mathbb{R}^{d}$ whose sum of degrees is $n$, where now a joint is a point $a$ incident to at least $d$ curves whose tangents at $a$ do not all lie in a common hyperplane.

In a rather surprising turn of events, the same results were obtained independently and simultaneously ${ }^{1}$ by Quilodrán [10]. The main ingredient of the proof is the same in both papers, but we believe that the peripheral part of the analysis is simpler in our paper.

\footnotetext{
${ }^{1}$ Both papers, Quilodrán's and ours, were posted on arXiv on the same day, June 2, 2009.
} 
We also note that this paper does not subsume the previous paper [6], because the new proof technique cannot handle the problem of counting incidences between lines and joints, nor can it handle flat points. Nevertheless, it is our hope that these extensions would also be amenable to similarly simpler proof techniques.

Analysis We will need the following well-known result from algebraic geometry; see proofs for the 3-dimensional case in $[6,9]$. We include the easy general proof for the sake of completeness.

Proposition 2 Given a set $S$ of $m$ points in $d$-space, there exists a nontrivial $d$ variate polynomial $p\left(x_{1}, \ldots, x_{d}\right)$ which vanishes at all the points of $S$, whose degree is at most the smallest integer $b$ satisfying $\left(\begin{array}{c}b+d \\ d\end{array}\right)>m$.

Proof A $d$-variate polynomial of degree $b$ has $\left(\begin{array}{c}b+d \\ d\end{array}\right)$ monomials, and requiring it to vanish at $m<\left(\begin{array}{c}b+d \\ d\end{array}\right)$ points yields $m$ linear homogeneous equations in the coefficients of these monomials. Such an underdetermined system always has a nontrivial solution.

Proof of Theorem 1 We only need to prove the upper bound. Let $L$ be a set of $n$ lines in $\mathbb{R}^{d}$, and let $J$ denote the set of joints of $L$. Put $m=|J|$, and assume to the contrary that $m>A n^{d /(d-1)}$, for some constant parameter $A$, depending on $d$, which we will fix shortly.

Pruning We first apply the following iterative pruning process to $L$. As long as there exists a line $\ell \in L$ incident to fewer than $m /(2 n)$ points of $J$, we remove $\ell$ from $L$, remove its incident points from $J$, and repeat this step with respect to the reduced sets of lines and points (keeping the threshold $m /(2 n)$ fixed). In this process we delete at most $m / 2$ points. We are thus left with a subset of the original lines, each incident to at least $m /(2 n)$ surviving points, and each surviving point is a joint in the set of surviving lines, that is, it is incident to at least $d$ surviving lines, not all in a common hyperplane. For simplicity, we continue to denote these sets as $L$ and $J$.

Vanishing Applying Proposition 2, we obtain a nontrivial $d$-variate polynomial $p$ which vanishes at all the (at most) $m$ points of $J$, whose degree is at most the smallest integer $b$ satisfying $\left(\begin{array}{c}b+d \\ d\end{array}\right) \geq m+1$, so the degree is at most

$$
b \leq\left\lceil(d ! m)^{1 / d}\right\rceil \leq 2(d ! m)^{1 / d} .
$$

We choose $A$ so that the number of points on each (surviving) line is greater than $b$. That is, we require that $m /(2 n)>2(d ! m)^{1 / d}$, or that $m>\left(4^{d} d !\right)^{1 /(d-1)} n$, which will hold if we choose $A>\left(4^{d} d !\right)^{1 /(d-1)}$. (Asymptotically, the right-hand side approaches $4 d / e$ as $d$ grows.)

With this choice of $A$, the polynomial $p$ vanishes on at least $m /(2 n)>b$ points on each line in $L$. Hence, $p$ vanishes identically on every line of $L$. 
Differentiating Fix a point $a \in J$, and let $\ell$ be a line of $L$ incident to $a$. Parameterize points on $\ell$ as $a+t v$, where $v$ is a (unit) vector in the direction of $\ell$. We have, for $t$ sufficiently small,

$$
p(a+t v)=p(a)+t \nabla p(a) \cdot v+O\left(t^{2}\right) .
$$

Since $p \equiv 0$ on $\ell$, we must have $\nabla p(a) \cdot v=0$. This holds for every line of $L$ incident to $a$. But since $a$ is a joint, the directions of these lines span the entire $d$-space, so $\nabla p(a)$, being orthogonal to all of them, must be the zero vector. That is, all the firstorder derivatives of $p$ vanish at $a$.

Consider one of these derivatives, say $p_{x_{1}}$, whose degree is at most $b-1$, and consider a line $\ell \in L$. Since $\ell$ contains more than $b-1$ points of $J$, and $p_{x_{1}}$ vanishes at each of these points, $p_{x_{1}}$ must vanish identically on $\ell$. That is, all first-order derivatives of $p$ vanish on all the lines of $L$.

We now repeat the above analysis to each of these derivatives. Consider again $p_{x_{1}}$, say, and pick a point $a \in J$ and an incident line $\ell \in L$. Using the above notation, we have, in complete analogy,

$$
p_{x_{1}}(a+t v)=p_{x_{1}}(a)+t \nabla p_{x_{1}}(a) \cdot v+O\left(t^{2}\right),
$$

for $t$ small enough, implying that $\nabla p_{x_{1}}(a)$ is orthogonal to each of the lines incident to $a$, and therefore must be zero. Applying this argument to each of the first-order derivatives, we conclude that all second-order derivatives of $p$ vanish at every point of $J$. Since the degree of these derivatives is at most $b-2$, they all vanish identically on every line of $L$.

Iterating this process, we conclude that all partial derivatives of $p$ vanish identically on all the lines of $L$. This however is impossible, because eventually we reach derivatives which are nonzero constants on $\mathbb{R}^{d}$. This completes the proof of the theorem.

\section{Extensions}

(1) Theorem 1 extends in a straightforward manner to the case of joints generated by algebraic curves in $\mathbb{R}^{d}$. Let $F_{i}(t)=\left(x_{1}^{(i)}(t), x_{2}^{(i)}(t), \ldots, x_{d}^{(i)}(t)\right)$ be an algebraic curve of degree $n_{i}$, and put $n=\sum_{i} n_{i}$. Define a joint to be a point $a$ incident to at least $d$ curves, such that the tangents at $a$ to the curves incident to $a$ are not all in a common hyperplane. Then the number $m$ of joints is at most $A n^{d /(d-1)}$, with the same constant $A$ as in the case of lines. Indeed, in an initial pruning step we get rid of those curves $F_{i}$ which pass through fewer than $m n_{i} /(2 n)$ joints, and of the joints lying on these curves. We lose in this pruning at most $m / 2$ joints. We then construct, as before, a polynomial $p$, of degree $b \approx m^{1 / d}$, which vanishes on all the surviving joints. Each of the surviving curves $F_{j}$ contains at least $m n_{j} /(2 n)$ surviving joints, and this quantity is larger than $b n_{j}$, by the initial assumption as in the preceding proof. Since $p$ vanishes at all these points, it follows from Bézout's theorem [4] that $p \equiv 0$ on each $F_{j}$. The proof then continues exactly as before, showing that all partial derivatives of $p$, of any order, vanish on all the surviving curves $F_{j}$, which yields a contradiction, as above. 
(2) One can also derive the following variant of Theorem 1: Let $L$ be a set of $n$ lines in $\mathbb{R}^{d}$, and let $2 \leq s<d$ be an integer. Call a point $a$ an $s$-joint if the affine hull of the directions of the lines of $L$ incident to $a$ is (at least) $s$-dimensional. Then the number of $s$-joints of $L$ is at most $A_{s} n^{s /(s-1)}$, where $A_{s}$ is the constant in Theorem 1 for dimension $s$. For a proof, simply project $L$ and the $s$-joints onto some generic $s$-flat ( $s$-dimensional affine subspace), and apply Theorem 1 to the projected lines and points.

However, as shown in [6] for the 3-dimensional case, the number of lines of $L$ incident to a point is also an important parameter, and not just the dimension of the flat that they span. In analogy with the analysis in [6], one could define a $(k, s)$-joint of $L$ to be a point $a$ incident to at least $k$ lines of $L$, which span a flat of dimension at least $s$, and ask for a bound on the number of $(k, s)$-joints, under some further restrictions on $L$. For example, it is shown in [6] that the number of $(3,2)$-joints in $\mathbb{R}^{3}$ is $O\left(n^{3 / 2}\right)$, provided that no plane contains more than $O(n)(3,2)$-joints.

(3) Singular points of spatial algebraic curves We finally state the following grand generalization of the above results. Let $C$ be a reduced algebraic curve (i.e., a curve with no multiple components) in the complex projective space $\mathbb{C P}^{d}$, for $d \geq 3$. The embedding dimension of $C$ at a point $a \in C$ is the smallest integer $s \geq 1$ such that there exists an analytic diffeomorphism $\varphi: U \rightarrow V$ of neighborhoods $U$ and $V$ of $a$ in $\mathbb{C P}^{d}$, so that $\varphi(C \cap U)$ is contained in an $s$-dimensional subspace of $\mathbb{C P}^{d}$ (cf. [8, Definition I.1.19 and Lemma I.1.24]). Clearly, the embedding dimension of a curve $C$ at any of its non-singular points is 1 , and the embedding dimension of a line configuration or of a configuration of curves, as above, at a joint (respectively, at an $s$-joint) is $d$ (resp., $s$ ). We then have:

Theorem 3 The number of points, where a reduced algebraic curve $C \subset \mathbb{C P}^{d}$ of degree $n$ has embedding dimension $s(s \geq 2)$ does not exceed $A_{s} n^{s /(s-1)}$, where $A_{s}$ is the constant from the proof of Theorem 1 for dimension $s$.

Indeed, the case $s=d$ (generalized joints) is treated precisely as in the proof of Theorem 1, since a hypersurface containing the curve $C$ must be singular at its generalized joints. In the case $s<d$ we apply a generic projection onto $\mathbb{C P}^{s}$, similar to the argument given in (2) above.

Acknowledgements The authors thank Shakhar Smorodinsky and Roel Apfelbaum for helpful discussions on this problem. In particular, Shakhar observed independently that the analysis also carries over to algebraic curves.

\section{References}

1. Bennett, J., Carbery, A., Tao, T.: On the multilinear restriction and Kakeya conjectures, 12 September 2005. arXiv:math/0509262

2. Brass, P., Moser, W., Pach, J.: Research Problems in Discrete Geometry. Springer, New York (2005)

3. Chazelle, B., Edelsbrunner, H., Guibas, L., Pollack, R., Seidel, R., Sharir, M., Snoeyink, J.: Counting and cutting cycles of lines and rods in space. Comput. Geom. Theory Appl. 1, 305-323 (1992)

4. Cox, D., Little, J., O’Shea, D.: Using Algebraic Geometry. Springer, Heidelberg (1998) 
5. Dvir, Z.: On the size of Kakeya sets in finite fields. J. Am. Math. Soc. 22, 1093-1097 (2009)

6. Elekes, Gy., Kaplan, H., Sharir, M.: On lines, joints, and incidences in three dimensions, manuscript, 11 May 2009. arXiv:0905.1583

7. Feldman, S., Sharir, M.: An improved bound for joints in arrangements of lines in space. Discrete Comput. Geom. 33, 307-320 (2005)

8. Greuel, G.-M., Lossen, C., Shustin, E.: Introduction to Singularities and Deformations. Springer, Berlin (2007)

9. Guth, L., Katz, N.H.: Algebraic methods in discrete analogs of the Kakeya problem, 4 December 2008. arXiv:0812.1043

10. Quilodrán, R.: The joints problem in $\mathbb{R}^{n}, 2$ June 2009. arXiv:0906.0555

11. Sharir, M.: On joints in arrangements of lines in space and related problems. J. Comb. Theory, Ser. A 67, 89-99 (1994)

12. Wolff, T.H.: Recent work connected with the Kakeya problem. In: Prospects in Mathematics, Princeton, NJ, 1996, pp. 129-162. Am. Math. Soc., Providence (1999) 